\title{
Untersuchung eines Falles von halbseitiger Farbensinnstörung am linken Ange.
}

(Aus dem physiolog. Institute der dentschen Universität in Prag.)

\author{
Von \\ Dr. Carl Hess, \\ Assistenten am Institute.
}

Die ausführliche Mittheilung des Falles, welcher den Gegenstand der vorliegenden Untersuchung bildet, ist wohl genügend gerechtfertigt durch das Interesse, das derselbe in klinischer sowohl als insbesondere in physiologischer Hinsicht bietet. In klinischer Hinsicht, weil Fälle von halbseitiger, nur auf ein Auge beschränkter Farbensinnstörung überhaupt verbältnissmässig selten zur Beobachtung kommen, in physiologischer, weil eingehendere Untersuchungen einer solchen Störung kaum vorliegen und weil unser Patient mit Hülfe von Methoden untersucht werden konnte, welche bis dahin in ähnlichen Fällen noch nicht zur Anwendung gekommen sind und welche es gestatten, ein klareres Bild von der Art und Weise der Abnahme des Farbensinnes zu gewinnen, als dies bisher möglich war.

Herr Professor Sattler hatte die grosse Freundlichkeit uns den Fall zu überlassen; bei der Ausführung der Untersuchungen hat mich Herr Professor Hering jederzeit mit Rath und That unterstützt. Es ist mir ein Bedürfniss, meinen verehrten Lehrern für die mir erwiesene Güte meinen aufrichtigen Dank zu sagen. 
Herr S., ein 31 jähriger Techniker, war früher stets gesund und hat bis vor zwei Monaten gut gesehen. Seit dieser Zeit macht sich eine Abnahme des Sehvermögens am linken Auge bemerkbar. Patient klagt, dass er die Gegenstände weniger scharf, die Farben nicht mehr so schön sehe wie früher. Insbesondere sei dies bei den grünen und rothen Farben auffällig.

Status praesens. Beiderseits Myopie $=4,0 \mathrm{D}$. Mit corrigirenden Gläsern ist die Sehschärfe des rechten Anges $={ }^{6} / 6$, jene des linken $={ }^{6} / 12$. Bei der ophthalmoskopischen Untersuchung findet sich eine schmale Sichel anf der temporalen Papillenhälfte. Die Papille selbst zeigt die gewöhnliche Färbung, die Netzhautgefässe sind normal, am übrigen Augenhintergrunde sieht man keinerlei krankhafte Veränderungen. Die Pupille des linken Auges ist etwas weiter als jene des rechten und zeigt ausgesprochenen Hippus.

Das rechte Ange ist in jeder Hinsicht vollständig normal.

Von anderweiten krankhaften Störungen bei dem Patienten ist nur das fast vollständige Fehlen des Patellarreflexes zu erwähnen.

Untersuchung mit Pigmenten.

Eine vorläufige Untersuchung zur Orientirung über den Farbensinn der verschiedenen Netzhautstellen bei dem Patienten wurde in der folgenden Weise vorgenommen. P. blickte bei fixirtem vorwärts geneigtem Kopfe aus einer Entfernung von $43 \mathrm{~cm}$ von oben auf eine grosse mit einem gleichmässig schwarzen Tuche überspannte horizontale Fläche. Senkrecht unter dem zu untersuchenden Auge befand sich eine kleine Marke als Fixationsobject. Während Patient diese (bei geschlossenem gesundem Auge) fixirte, wurden farbige Scheiben von $3 \mathrm{~cm}$ Durchmesser erst an die Stelle des directen Sehens, dann auf verschiedenen Meridianen an etwas indirect gesehene Stellen des Sehfeldes gebracht. Dabei zeigte sich, dass auf allen Stellen der nasalen Sehfeldhälfte die Scheiben in der ,richtigen" Farbe erschienen, d. h. so, wie dem normalen Auge auf den entsprechenden Stellen, während auf allen Stellen der tomporalen Sehfeldhälfte sämmtliche Pigmente mehr oder weniger 
deutlich verändert gesehen wurden, auch wenn die Scheiben bis dicht an den Fixationspunkt herangeschoben waren. Die Trennungslinie zwischen den beiden Hälften fiel annähernd mit der vertikalen Trennungslinie der Netzhaut zusammen, bis auf die Stelle des directen Sehens, welche ganz in das erkrankte Gebiet einbezogen war. An diese Stelle schloss sich nasalwärts unmittelbar gesundes Netzhautgebiet an. Wenn also Patient Objecte von nicht allzu geringem Durchmesser fixirte, so konnte leicht ihr Bild, zum Theile wenigstens, auf gesunde Netzhaut fallen. Zur ausschliesslichen Untersuchung des Farbensinns der erkrankten Parthieen musste man also entweder sehr kleine Objecte verwenden, deren Bild auch bei directer Fixation nur auf kranke Parthieen fiel, was indessen aus verschiedenen Gründen unzweckmässig erschien, oder man musste bei Benutzung grösserer Objecte den Patienten diese etwas excentrisch betrachten lassen, so dass ihr Bild auf der nasalen Netzhauthälfte entworfen wurde.

Die folgende Zusammenstellung giebt eine Uebersicht über die Aenderungen des Tones und der Sättigung, welche die mit den nasalen Netzhautparthieen gesehenen Pigmentlichter erfuhren. Es wurde dabei so verfahren, dass, während Patient die Marke auf dem schwarzen Grunde fixirte, eine farbige Scheibe auf eine $2 \mathrm{~cm}$ nasalwärts, eine zweite ganz gleiche Scheibe auf eine ebensoweit temporalwärts gelegene Stelle des Grundes gelegt wurde. Patient sah jetzt die erstere immer in ihrer ,richtigen" Farbe (d.i. so wie mit seinem gesunden Auge), die andere mehr oder weniger verschieden, und zwar in der folgenden Weise:

Sattes Spectralroth erschien "schmutzig dunkelgelb“,

Sattes Orange erschien "schmutzig schwefelgelb",

Sattes Chromgelb "Grelb, matter als die andere Scheibe“, Sattes Gelbgrün erschien „schwach gelblichgrau“, Ein dem Urgrün nahestehendes ziemlich gesättigtes Grün erschien "grünlichgrau", 
Untersuchung eines Falles von halbseitiger Farbensinnstörung. 27

Sattes Blau „Blau, etwas matter als die andere Scheibe", (mit einem „violetten“ Tone),

Sattes Violett erschien ,violett, aber mehr bläulich als die andere Scheibe",

Sattes Blauroth "grauviolett"

Weiss, Hellgrau und Dunkelgrau wurden auf beiden Seiten gleich gesehen.

Herstellung von Gleichungen mit Pigmentlichtern.

Um einen genaueren Aufschluss über die Art der Aenderungen zu erhalten, welche die mit der kranken Netzhauthälfte gesehenen farbigen Lichter erfuhren, insbesondere, um ron den rom Patienten gebrauchten Benennungen dieser Lichter unabbängig zu sein, wurden nun Gleichungen hergestellt zwischen zwei Lichtern, von welchen das eine auf der erkrankten, das andere auf der gesunden Netzhauthälfte zur Wirkung kam, beide aber auf einer nur wenige Grade von der Fovea centralis entfernten, also beim gesunden Auge noch vollkommen farbentüchtigen Stelle. Das hierbei eingeschlagene Verfahren war das folgende:

Patient blickte aus einer Entfernung von $30 \mathrm{~cm}$ auf eine gleichmässig graue Fläche, in welcher zwei kreisrunde Löcher von $3 \mathrm{~cm}$ Durchmesser so ausgeschlagen waren, dass ihre medialen Ränder $3 \mathrm{~cm}$ von einander abstanden. In der Mitte zwischen beiden Löchern befand sich ein feiner Fixationspunkt. Es bildete sich demnach, wenn das Auge diesen fest fixirte, was rom Patienten stets gewissenhaft befolgt wurde, von den Löchern das eine auf einer ca. $5^{0}$ temporalwärts, das andere auf einer ebensoweit nasalwärts gelegenen, jedes also auf einer beim Gesunden noch vollkommen farbentüchtigen extramacularen Netzhautstelle ab. Unterhalb dieser Löcher liessen sich ganz in der gleichen Weise, wie an dem in der Abhandlung von Herrn Professor Hering ${ }^{1}$ ) beschriebenen Apparate zur Herstellung binocu-

1) Vgl. Hering. Dieses Archiv Bd. XXXVI, 3, S. 1. 
larer Farbengleichungen verschiedene farbige Platten anbringen, welchen farbloses oder durch bunte Gläser gefärbtes Licht durch Spiegelung zugemischt werden konnte. Durch das rechte Loch in der grauen Fläche war die rechte Platte sichtbar, durch das linke Loch die linke Platte; beide erschienen als farbige Kreisflecke in der Fläche; einem jeden der beiden konnte jede beliebige Farbe gegeben werden. (Ueber die Details vergl. die erwähnte Abhandlung von Hering.)

In der folgenden Tabelle sind die auf diesem Wege hergestellten Gleichungen angegeben. In der ersten Columne sind die Lichter verzeichnet, welche auf der kranken Netzhauthälfte zur Wirkung kamen, in der zweiten jene, deren Bild auf die gesunde Netzhauthälfte fiel; bei den letzteren ist zugleich angegeben, in welcher Weise dieselben hergestellt wurden. Dem gesunden Auge des Patienten erschienen die beiden Löcher, wenn für das kranke die Gleichung hergestellt war, ganz verschieden, und zwar ebenso wie uns.

Tabelle der Gleichungen zwischen zwei Lichtern, von welchen das eine auf der gesunden, das andere auf der kranken Netzhauthälfte zur Wirkung kam.

\begin{tabular}{c|l}
\hline Kranke Netzhauthälfte & \multicolumn{1}{|c}{ Gesunde Netzhauthälfte } \\
\hline \hline $\begin{array}{c}\text { 1) Sattes } \\
\text { Spectralroth. }\end{array}$ & $\begin{array}{l}\text { Dunkles, wenig gesättigtes Gelb, mit } \\
\text { einem leichtem Stich ins olivenfarbige, herge- } \\
\text { stellt durch eine chromgelbe Platte, welche } \\
\text { stark vom einfallenden Lichte weggeneigt war, } \\
\text { und der noch weisses Licht zugespiegelt wurde. }\end{array}$ \\
2) Sattes Orange. & $\begin{array}{l}\text { Wenig gesăttigtes Gelb, mit leichtem Stich } \\
\text { ins Olivenfarbige, hergestellt in ähnlicher Weise } \\
\text { wie das vorige. } \\
\text { Weissliches Gelb, hergestellt durch Zu- } \\
\text { 3) Gesättigtes } \\
\text { Chromgelb. } \\
\text { mischung weissen Lichtes zur chromgelben } \\
\text { Platte und leichtes Neigen derselben vom ein- } \\
\text { fallenden Lichte weg. }\end{array}$
\end{tabular}


Untersuchung eines Falles von halbseitiger Farbensinnstörung. 29

\begin{tabular}{|c|c|}
\hline Kranke Netzhanthälfte & Gesunde Netzhauthälfte. \\
\hline $\begin{array}{l}\text { 4) Gesättigtes } \\
\text { Gelbgrün. }\end{array}$ & $\begin{array}{l}\text { Leicht grünliches Graugelb, hergestellt } \\
\text { durch Zumischung weissen Lichtes zur gelb- } \\
\text { grünen Platte und leichte Neigung derselben } \\
\text { vom einfallenden Lichte weg. }\end{array}$ \\
\hline $\begin{array}{l}\text { 5) Ein dem Ur- } \\
\text { grün naheste- } \\
\text { hendes gesät- } \\
\text { tigtes Grün. }\end{array}$ & $\begin{array}{l}\text { Schwach grünliches Grau, hergestellt durch } \\
\text { Zumischung von viel weissem lichte zur grü- } \\
\text { nen Platte und Neigung derselben vom einfal- } \\
\text { lenden Lichte weg. }\end{array}$ \\
\hline $\begin{array}{l}\text { 6) Gesättigtes } \\
\text { Blau. }\end{array}$ & $\begin{array}{l}\text { Weisslichesweniggesättigtes Blau, her- } \\
\text { gestellt durch Zumischung von weissem Lichte } \\
\text { zur blauen Platte und leichte Neigung dersel- } \\
\text { ben vom einfallenden Lichte weg. }\end{array}$ \\
\hline $\begin{array}{l}\text { 7) Gesätigtes } \\
\text { Violett. }\end{array}$ & $\begin{array}{l}\text { Wenig gesättigtes Blau, in ähnlicher Weise } \\
\text { hergestellt wie das vorige. }\end{array}$ \\
\hline $\begin{array}{l}\text { 8) Helles Blau- } \\
\text { roth. }\end{array}$ & $\begin{array}{l}\text { Weniggesättigtes Blau, in ähnlicher Weise } \\
\text { hergestellt wie das vorige. }\end{array}$ \\
\hline
\end{tabular}

War beiderseits ein Grau von gleicher Helligkeit eingestellt, welches jedoch heller war, als das Grau der oberen Fläche, so erschien dem Patienten das mit der kranken Netzhauthälfte gesehene Loch um ein Geringes minder hell als das andere. Durch eine leichte Neigung der betreffenden Platte zum einfallenden Lichte wurde die Gleichung zwischen beiden Löchern hergestellt. Uns erschien dieses Loch jetzt um ein Weniges heller als das andere. War unten beiderseits ein dunkleres Grau eingestellt, so erschien das mit der kranken Netzhauthälfte gesehene Loch etwas weniger dunkel. Die Unterschiedsempfindlichkeit für Helligkeiten war auf der kranken Netzhauthälfte etwas kleiner als auf der gesunden.

Untersuchung mit homogenen Lichtern. Das Gesichtsfeld eines kleinen Fernrohres, welches mit einem Spectralapparate verbunden war $^{1}$ ), wurde mit homogenem

1) Ueber die Einrichtung des Apparates vergl. Hering: Ueber individuelle Verschiedenheiten des Farbensinnes Seite 20. 
Lichte erfüllt; Patient fixirte dieses kreisförmige Feld, dessen scheinbarer Durchmesser auf eine Entfernung von $30 \mathrm{~cm}$ projicirt, $3 \mathrm{~cm}$ betrug. Es wurden der Reihe nach Lichter verschiedener Wellenlänge eingestellt. Als Lichtquelle diente das von einem Spiegel reflectirte Tageslicht. Die Spaltbreite war für alle Lichter die gleiche.

Patient bezeichnet ein Licht von der mittlen Wellenlänge $690 \mu \mu$ (für uns ein sehr dunkles Roth) als Gelb, ein Licht von der Wellenlänge 656,2 $\mu \mu$ (Linie C) als Orangegelb, ein Licht von der Wellenlänge $620 \mu \mu$ als Schwefelgelb. Alle Lichter von der Wellenlänge $620 \mu \mu$ bis ungefähr $525 \mu \mu$ als mehr oder weniger schönes Gelb, Lichter von ungefähr $525 \mu \mu$ bis $500 \mu \mu$ als grünliches Gelb.

Ein Licht von der Wellenlänge $495 \mu \mu$ wird als schwach grünlich, bezw. als leicht grünliches Grau bezeichnet; es erscheint "weder gelblich noch bläulich“. Licht von der Wellenlänge $496 \mu \mu$ und mehr wird regelmässig als schwach gelblich, Licht von der Wellenlänge $494 \mu \mu$ und weniger als schwach bläulich bezeichnet.

Alle Lichter von der Wellenlänge $494 \mu \mu$ bis ungefähr $458 \mu \mu$ werden mehr oder weniger deutlich blau, ein der Linie $G$ entsprechendes Licht blau „mit schwach violettem Hauch" gesehen.

Die Untersuchung am continuirlichen Spectrum ergab, dass dasselbe für das kranke Auge, mit unserem Auge verglichen, am rothen Ende kaum merklich, am violetten Ende gar nicht verkürat erschien.

Bei diesen Beobachtungen hatten wir indessen nicht die volle Sicherheit, dass das homogene Licht wirklich ausschliesslich auf der erkrankten Netzhaut zur Wirkung kam. (vergl. oben S. 26). Es war daher nothwendig, die Untersuchung auch in der Weise anzustellen, dass eine Wirkung der betreffenden Lichter auf die gesunden Netzhautstellen sicher ausgeschlossen war.

Um Gleichungen zwischen farbigen Lichtern, welche 
ausschliesslich auf der nasalen und solchen, die auf der temporalen Netzhauthälfte zur Wirkung kamen, auch mit homogenen Farben herstellen zu können, wurde die folgende Einrichtung getroffen.

In dem Fernrohre des erwähnten Apparates wurde an passender Stelle ein kreisförmiges Diaphragma angebracht, welches durch einen von oben nach unten gehenden breiten Steg in zwei seitliche Hälften getheilt war. Der durch das Fernrohr blickende Beobachter sah also zwei durch einen breiten dunklen Streifen von einander getrennte nahezu halbkreisförmige Felder. Die scheinbare Breite des trennenden Steges betrug, auf eine Entfernung von $30 \mathrm{~cm}$ projicirt $3 \mathrm{~cm}$, die seitlichen Gesichtsfeldhälften hatten eine scheinbare Höhe von fast $7 \mathrm{~cm}$ und (an der breitesten Stelle) eine scheinbare Breite von $2 \mathrm{~cm}$. In der Mitte des Streifens war ein kleines Loch angebracht, durch welches etwas von dem spectralen Lichte drang und welches als Fixationspunkt diente. Von den beiden leuchtenden Feldern bildete sich demnach, wenn das Auge auf diesen Punkt gerichtet. war, jedes auf einer von der Fovea centralis im horizontalen Meridian ca. $5^{\circ}$ entfernten Netzhautstelle ab. Jede der beiden Hälften konnte für sich mit beliebigem homogenen Lichte erfüllt werden; der rechten (also bei Betrachtung mit dem erkrankten linken Auge des Patienten auf dessen temporaler, gesunder Netzhauthälfte sich abbildenden) Gesichtshälfte konnte ausserdem noch farbloses, von einer mattgeschliffenen Milchglasplatte reflectirtes Tageslicht in beliebiger Menge zugemischt, die Helligkeit eines jeden der beiden Felder durch Regulirung der Breite des zugehörigen Spaltes beliebig variirt werden. Die mittlen Wellenlängen der jeweils eingestellten Lichter waren jederzeit genau zu bestimmen.

Es wurde nun zunächst auf der rechten (nasalen) Gesichtshälfte, (welche also mit der gesunden Netzhauthälfte gesehen wurde), homogenes reines Gelb von der Wellen- 
länge $574,5 \mu \mu$ eingestellt. Die temporale Gesichtsfeldhälfte wurde der Reihe nach mit verschiedenen Lichtern grösserer Wellenlänge erfüllt.

War in der letzteren ein Licht von der Wellenlänge 656,2 $\mu \mu$ (Linie C) eingestellt, so erschien dem Patienten diese Hälfte „schmutzig gelb“, die rechte schön gelb. Durch Zumischung farblosen Lichtes zu der letzteren und durch entsprechende Herabsetzung der Helligkeit des homogenen Lichtes liess sich eine Gleichung zwischen beiden Hälften herstellen. Dẹm gesunden Auge erschien jetzt die linke Gesichtsfeldbälfte schön roth, die rechte schmutzig gelb.

In ähnlicher Weise wurde eine Gleichung zwischen einem gelblich rothen Lichte von der Wellenlänge $610 \mu \mu$ und einem weisslich-gelben, endlich zwischen einem der Linie D $(589,7 \mu \mu)$ entsprechenden und einem wenig gesättigten gelben Lichte hergestellt.

Wurde die linke Gesichtsfeldhälfte mit gelbgrünem Lichte von der Wellenlänge $551 \mu \mu$ oder mit einem Lichte aus der Gegend zwischen den Linien $\mathrm{E}$ und $\mathrm{b}$ des Spectrums erfüllt, so konnte wiederum durch Zumischung farblosen Lichtes zur rechten, mit homogenem Gelb von $574,5 \mu \mu$ Wellenlänge erfüllten Hälfte und durch entsprechende Aenderung der Breite des Spaltes für dieses Licht eine Gleichung zwischen beiden Gesichtsfeldhälften hergestellt werden. Dem gesunden Auge erschien jetzt die rechte Gesichtsfeldhälfte als ein blasses Gelb, (die linke als schönes Grüngelb, bezw. gelbliches Grün).

Wurden beide Gesichtsfeldhälften mit homogenem reinem Gelb von der Wellenlänge $574,5 \mu \mu$ erfüllt, so sah $\mathrm{Pa}-$ tient die linke Hälfte gelb, aber "nicht so rein" wie die rechte. Durch Zumischung von farblosem Lichte $\mathrm{zu}$ dieser letzteren und entsprechende Aenderung der Spaltbreite für das homogene Licht wurde leicht eine Gleichung erhalten. Dem gesunden Auge erschien jetzt die rechte Hälfte „matt gelb“ im Vergleich zum gesättigten Gelb der linken Hälfte. 
Untersuchung eines Falles von halbseitiger Farbensinnstörung. 33

Eine zweite Reihe von Gleichungen wurde hergestellt, indem die rechte Gesichtsfeldhälfte mit homogenem reinem Blau (von der Wellenlänge $471 \mu \mu$ ) die andere der Reihe nach mit grünblauem, blauem und violettem Lichte erfüllt wurde. War auf der linken Hälfte ein Grünblau von der. Wellenlänge $485 \mu \mu$ eingestellt, so liess sich durch $\mathrm{Zu}$ mischung farblosen Lichtes zum reinen Blau der anderen Seite und durch Regulirung der Spaltbreite für dieses Licht eine Gleichung herstellen. Das gesunde Auge sah dieses jetzt als ein sehr weissliches Blau. War die linke Gesichtsfeldhälfte mit homogenem Violett (Wellenlänge etwa $435 \mu \mu)$ erfüllt, so erschien jetzt dem Patienten diese letztere "gesättigter blau" als die rechte Hälfte. Da unser Apparat es nicht gestattete, diesem Violett farbloses Licht zuzumischen, so musste die zur Herstellung einer Gleichung nothwendige Sättigungsminderung des homogenen Violett durch Zumischung gelben Lichtes bewerkstelligt werden. Wir benutzten anstatt des reinen Gelb ein etwas ins Rothe gehendes Gelb. Für den vorliegenden Versuch war dies ganz gleichgültig, weil das benutzte rothgelbe Licht für die kranke Netzhauthälfte des Patienten keine rothe Valenz besass (s. o.). Es liess sich auf diese Weise eine Gleichung zwischen homogenem Blau, das auf der gesunden Netzhauthälfte, und einem aus homogenem Violett und Rothgelb gemischten röthlichen Violett, das auf der kranken Netzhauthälfte zur Wirkung kam, herstellen. (Dem gesunden Auge erschien das Rothviolett heller als das homogene Blau.)

Waren beide Gesichtsfeldhälften mit homogenem, reinem Blau von der Wellenlänge $471 \mu \mu$ erfüllt, so sah $\mathrm{Pa}$ tient beide blau, die linke Hälfte etwas matter. Die Gleichung zwisehen beiden wurde leicht durch Zumischung farblosen Lichtes zur rechten Hälfte erzielt; dem gesunden Auge erschien diese jetzt im Vergleich zur linken weisslich blau.

Bei einer dritten Versuchsreihe wurde die rechte Gesichtsfeldhälfte nur mit farblosem Tageslichte, welches von

v. Graefe's Archiv für Ophthalmologie. XXXVI. 3. 
der mattgeschliffenen Milchglasplatte reflectirt ward, erfüllt, die linke der Reihe nach mit Lichtern von der Wellenlänge $500 \mu \mu$ bis $490 \mu \mu$.

War ein Licht von der Wellenlänge $495 \mu \mu$ eingestellt, so konnte durch Regulirung der Helligkeit der mit farblosem Lichte erfüllten Gesichtsfeldhälfte eine genaue Gleichung zwischen dieser und der mit grünem Lichte erfüllten Hälfte hergestellt werden. Patient sah jetzt beide „rein grau“.

Es könnte auffällig erscheinen, dass Patient bei Bestimmung der "neutralen Stelle" im Spectrum, wenn das ganze Gesichtsfeld mit homogenem Lichte erfüllt war, das gleiche Licht einstellte, wie bei der Herstellung einer Gleichung zwischen dem Tageslichte und der ihm farblos erscheinenden Stelle des Spectrums, während ein von Geburt Rothgrünblinder in diesem Falle in der Regel ein Licht von etwas anderer Wellenlänge einstellt. Die nächstliegende Erklärung dafür dürfte wohl in dem Umstande zu suchen sein, dass auch das Vermögen der Blaugelbempindung bei dem Patienten etwas herabgesetzt war.

Die Perimeteruntersuchung an dem von mir in meiner Abhandlung "Ueber den Farbensinn beim indirecten Sehen" ${ }^{1}$ ) beschriebenen Hering'schen Apparate lieferte das folgende Ergebniss.

Das auf den Kreiselscheiben hergestellte unveränderliche Roth und Grün von gleicher farbiger und gleicher weisser Valenz wurde bei directer Fixation und auf der ganzen nasalen Netzhauthälfte nicht wahrgenommen. Das Loch in der oberen grauen Fläche erschien dem Patienten ganz gleich mit der grauen Fläche selbst, wenn ich der letzteren für mein Auge bei genügend indirectem Sehen die gleiche weisse Valenz mit dem rothen bezw. grünen Pigmente auf der Kreiselscheibe gegeben hatte. Auf der temporalen Netzhauthälfte wurde das Roth und Grün gut wahrgenommen, bei zunehmend indirectem Sehen wurden beide an der gleichen Stelle farblos. Die Grenzen der Wahrnehmbarkeit stimmten annähernd

1) Dieses Archiv Bd. XXXV, 4. Abth. 
genau mit den für mein Auge in den entsprechenden Halbmeridianen gefundenen Grenzen überein.

Ein unveränderliches Gelb und Blau von gleicher farbiger und gleicher weisser Valenz und von verhältnismässig geringer Sättigung wurden bei directem Sehen richtig benannt. Auf der temporalen Netzhauthälfte wurden beide bei zunehmend indirectem Sehen an der gleichen Stelle farblos; die gefundenen Grenzen waren die gleichen, wie für mein Auge. Auf der nasialen Netzhauthälfte wurde das Blau und das Gelb ebenfalls an der gleichen Stelle farblos und zwar auf dem horizontalen inneren Meridian schon bei einer Entfernung von $20-22^{\circ}$ von der fixirten Stelle, während für mein Auge auf dem gleichen Meridian die Grenzen sehr viel weiter excentrisch lagen. (Ich verzichtete auf eine genauere Bestimmung wegen allzu indirecten Sehens.)

Die Grenzen der Wahrnehmbarkeit für farbloses Licht waren auf der nasalen Netzhauthälfte des erkrankten Auges, verglichen mit jenen auf den entsprechenden Meridianen des gesunden Auges, gleichfalls nicht unbeträchtlich eingeschränkt. Genauere Messungen konnten nicht vorgenommen werden.

Das Ergebniss, welches die Untersuchung des vorliegenden Falles lieferte, lässt sich kurz zusammenfassen wie folgt:

An dem linken Auge des Patienten ist auf der nasalen Netzhauthälfte der Rothgrünsinn nahezu vollständig geschwunden, das Vermögen der Gelb- und das der Blauempfindung beträchtlich herabgesetzt und zwar haben beide in gleichem Maasse gelitten, wie aus der perimetrischen Untersuchung (Zusammenfallen der Grenzen für gleichwerthiges Gelb und Blau) hervorgeht. Das Vermögen der Schwarzweissempfindung hat gleichfalls, wenn auch sehr viel weniger gelitten. Patient sieht dementsprechend mit der erkrankten Netzhauthälfte rein rothe und grüne Pigmentlichter von nicht zu grosser Sättigung farblos, (hell- oder 
dunkelgrau), gelbe und blaue Lichter in dem gleichen Tone wie mit der gesunden Netzhauthälfte, nur minder gesättigt; farblose Lichter erscheinen auf der gesunden und der kranken Netzhauthälfte farblos und in ihrer Helligkeit nicht wesentlich verschieden. In den Zwischenfarben Orange, Gelbgrün, Blaugrün und Violett sieht Patient nur das Gelb, bezw. Blau.

Ein analoges Resultat ergiebt die Untersuchung mit homogenen Lichtern; Patient sieht mit der kranken Netzhauthälfte alle homogenen Lichter weniger gesättigt als mit der gesunden. Ein grünes Licht von der Wellenlänge $495 \mu \mu$ erscheint ihm farblos. ${ }^{1}$ ) Alle Lichter grösserer Wellenlänge sieht er gelb, alle Lichter kleinerer Wellenlänge blau. Ein Gelb von der mittleren Wellenlänge $574,5 \mu \mu$ und ein Blau von der mittleren Wellenlänge $471 \mu \mu$ erscheinen auf der kranken Netzhauthälfte in dem gleichen Tone, wie auf der gesunden, nur minder gesättigt, alle anderen Lichter zeigen ausser der Aenderung der Sättigung auch eine mehr oder minder deutliche Aenderung des Tonos.

Aus alledem geht hervor, dass die ganze nasale Netzhauthälfte des Patienten sich in Bezug auf den Farbensinn so verhält, wie eine bestimmte, ziemlich weit excentrisch gelegene Stelle eines normalen Auges.

Dieselben Gründe, welche eine Erklärung des Farbensinnes beim indirecten Sehen nach der Dreifasertheorie unmöglich erscheinen lassen, haben in gleicher Weise Geltung für den vorliegenden Fall.

Nach der Theorie der Gegenfarben erklärt sich dor Fall in der Weise, dass von den beiden farbigen Variabeln des Farbensinnes die roth-grüne fast ganz, die gelb-blaue zu einem Theile ausser Function gesetzt ist, während die weiss-schwarze nur wenig gelitten hat.

1) Dieses Licht ist genau das gleiche wie jenes, welches ich früher (vergl. dieses Arch. Bd. XXXV, 4, S. 20) für mein Auge als "unveränderliches Grün" bestimmt habe; ich fand damals $494-497 \mu \mu$. 